\title{
ANÁLISE NUMÉRICA CFD DA DISPERSÃO DE LÍQUIDO EM UM LAVADOR VENTURI DE SEÇÃO RETANGULAR.
}

\author{
V. K. HONDA ${ }^{1}$, R. BÉTTEGA ${ }^{1}$, J.A.S. GONÇALVES ${ }^{1}$ e V. G.GUERRA ${ }^{1}$ \\ ${ }^{1}$ Universidade Federal de São Carlos, Departamento de Engenharia Química \\ E-mail para contato: vadila@ufscar.br
}

\begin{abstract}
RESUMO - O lavador Venturi é um equipamento com alta eficiência na remoção de partículas de efluentes gasosos. O líquido ao entrar no lavador sofre atomização formando gotas, que atuam como coletoras das partículas. Uma distribuição uniforme do das gotas no lavador melhora o contato entre líquido e partícula, influenciando a eficiência de coleta. Desta forma, estudos acerca de condições operacionais considerando o uso apropriado de líquido e a sua distribuição no lavador, bem como modelos computacionais para sua previsão, são relevantes. Neste contexto, o presente estudo tem por objetivo avaliar numericamente a distribuição de líquido em um lavador Venturi para diferentes condições operacionais e comparar com resultados experimentais. O comportamento fluidodinâmico do escoamento ar-água foi simulado utilizando o pacote comercial ANSYS FLUENT 14.0 através da implementação de um modelo de trajetória para fase discreta. Os resultados simulados de dispersão de líquido mostraram concordância com os dados experimentais. Os resultados simulados indicaram alta dependência da resposta com o diâmetro médio que é alimentado no modelo de distribuição de tamanho de gotas.
\end{abstract}

\section{INTRODUÇÃO}

O controle da emissão de efluentes gasosos é de grande importância para a preservação do meio ambiente, assim, é de grande interesse que haja maneiras eficientes e econômicas de fazê-lo.

Dos equipamentos disponíveis o lavador do tipo Venturi é muito utilizado na limpeza de gases e controle de poluição. Isso se deve a sua alta eficiência na remoção de partículas numa ampla faixa granulométrica, além de ser compacto e possibilitar a reutilização do líquido de lavagem.

Desde os primeiros estudos em lavadores Venturi é consenso que a distribuição de concentração de gotas no interior do equipamento é uma variável de grande importância no seu desempenho (Ekman e Johnstone, 1951). Dessa forma, é de grande interesse conhecer detalhadamente o efeito da relação entre os parâmetros de projeto e a distribuição de gotas, com o intuito de aprimorar o desempenho e diminuir os custos operacionais do lavador. Dessa forma, o presente trabalho tem com objetivo avaliar a distribuição de gotas no interior de um lavador Venturi retangular para diferentes condições experimentais a partir de comparações entre dados experimentais e simulação CFD (Computational Fluid Dynamics). 


\subsection{Distribuição de Tamanho de Gotas}

O líquido de limpeza geralmente é injetado no lavador na constrição do lavador (garganta). Quando o líquido é introduzido na forma de jatos, ao interagir com a corrente gasosa ele é rapidamente transformado em um grande número de pequenas gotas que se distribuem no interior do sistema sob a influência do escoamento gasoso turbulento. As gotas se movem em ambas as direções, axial e radial. Algumas dessas gotas, quando alcançam a parede do equipamento, formam um filme que praticamente não contribui no processo de limpeza (Ananthanarayanan e Viswanathan, 1999).

Lavadores com má distribuição de gotas apresentam regiões altamente concentradas e regiões que apresentam baixa concentração de líquido, o que ocasiona menor desempenho em comparação com lavadores com boa distribuição de líquido, nos quais as gotas cobrem a região da garganta uniformemente (Gonçalves et al., 2004).

A dispersão de gotas é função de parâmetros de projeto como a razão líquido gás (L/G) e o número de orifícios de injeção de líquido e, consequentemente, também é função da penetração do jato líquido.

\section{MATERIAIS E MÉTODOS}

O equipamento utilizado nos testes experimentais foi um lavador Venturi constituído por módulos de acrílico que possui uma seção transversal retangular e uma garganta de dimensões de 27 x40 mm. A injeção de líquido foi realizada por uma bomba helicoidal que transportava água de um reservatório até o primeiro módulo da garganta do lavador, no qual havia quatro orifícios de injeção de $1 \mathrm{~mm}$ de diâmetro, um em cada parede da garganta. Um esquema do equipamento utilizado com as suas principais dimensões pode ser visto na Figura 1.

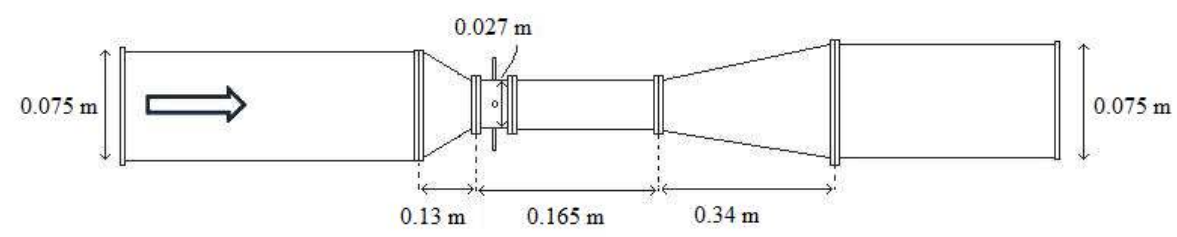

(a)

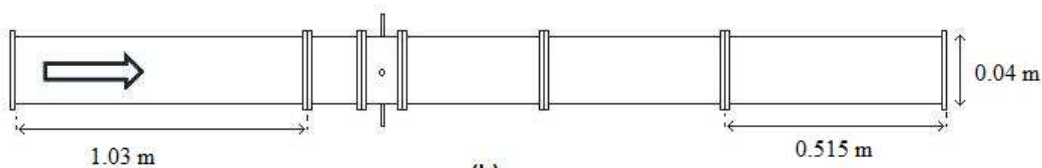

(b)

Figura 1 - Lavador Venturi em módulos utilizado nos testes experimentais.(a) vista superior (b) vista lateral.

A dispersão de gotas foi medida pelo método de amostragem isocinética, método utilizado com sucesso por Viswanathan et al. (1984) e por Gonçalves et al. (2004). Para se empregar esse método utilizou-se uma seção de teste em acrílico, acoplada entre a garganta e o difusor. Essa seção de teste 


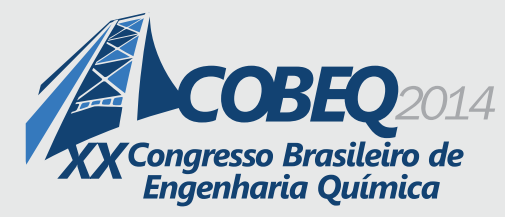

possuía uma sonda na qual ar e parte das gotas que escoam pela garganta são sugadas para um pequeno ciclone de alumínio contendo uma coluna de sílica gel em seu topo. A função do ciclone é separar a corrente bifásica e reter o líquido. A sucção foi realizada por uma bomba e ajustada por um medidor de vazão para que a velocidade de sucção fosse igual à velocidade do ar no interior da garganta. A Figura 2 apresenta a vista geral do experimento para amostragem das gotas.

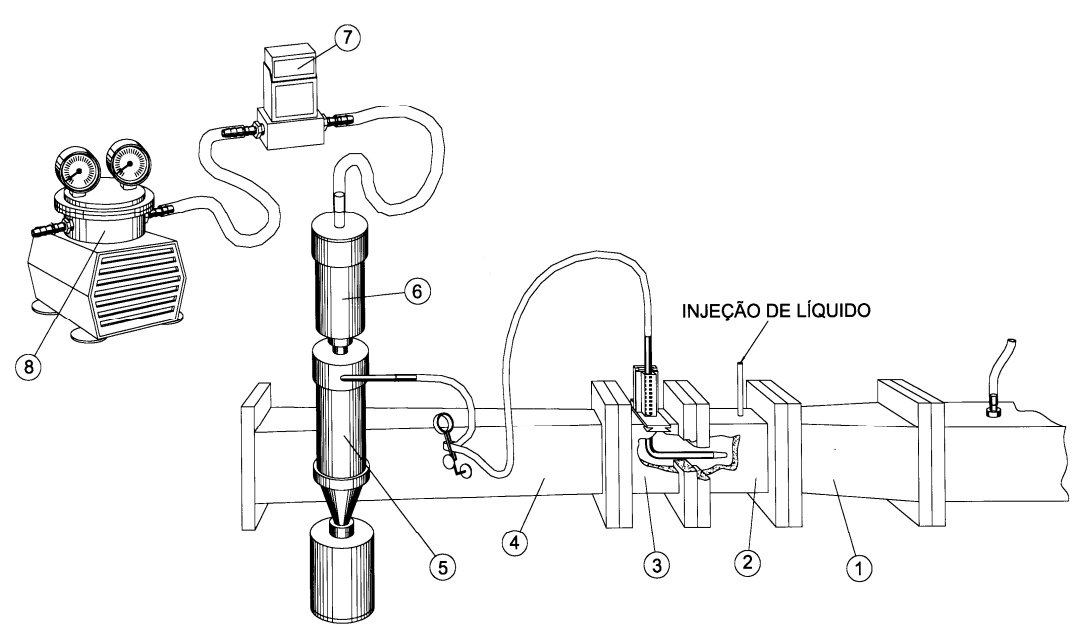

$$
\begin{aligned}
& 1 \text { - Seção convergente do lavador Venturi } \\
& 2 \text { - Primeira seção da garganta } \\
& \text { - Seção de inserção da sonda de amostragemm } \\
& \text { 4- Seção divergente do lavador Venturi } \\
& \text { 5- Ciclone para coleta de gotas } \\
& \text { 6- Coluna de sílica gel } \\
& \text { 7- Controlador de fluxo } \\
& \text { 8- Bomba de sucção }
\end{aligned}
$$

Figura 2 - Aparato experimental para medida da dispersão de gotas.

A fim de se efetuar a amostragem em várias posições sobre um mesmo plano transversal, a seção foi construída de forma que se possibilitasse o movimento na direção vertical e horizontal. Nos testes realizados optou-se por medir apenas os pontos centrais do plano, como pode ser visto na Figura 3. A dispersão de gotas no interior da garganta foi avaliada a uma distância de 1,6 cm dos orifícios de injeção de líquido. Os testes experimentais realizados estão apresentados na Tabela 1.

Tabela 1 - Condições experimentais avaliadas

\begin{tabular}{|c|c|c|c|}
\hline Experimento & Número de orifícios & $\mathrm{Q} 1(\mathrm{ml} / \mathrm{min})$ & $\mathrm{Vg}(\mathrm{m} / \mathrm{s})$ \\
\hline E-1 & 1 & 300 & 64 \\
\hline E-2 & 2 & 600 & 64 \\
\hline E-3 & 2 & 600 & 74 \\
\hline
\end{tabular}

A dispersão foi medida através do fluxo mássico de líquido recolhido em cada ponto do plano transversal mostrado na Figura 3.

A solução matemática do problema foi realizada utilizando-se o pacote comercial de CFD ANSYS FLUENT 14.0. Foram utilizados o modelo lagrangeano de fase discreta para prever a dispersão do líquido no equipamento. Muitos dos parâmetros do modelo foram estabelecidos com base no artigo de Ananthanrayanan e Viswanathan (1999), com exceção dos parâmetros de distribuição de tamanho de gotas. 




Figura 3 - Vista geral do equipamento para medida da dispersão de gotas.

A distribuição de tamanho de gotas foi obtida experimentalmente utilizando-se o equipamento Malvern Spraytec. O princípio de funcionamento desse equipamento é a difração de raios laser. O laser, quando incide sobre uma gota, faz com que parte da energia luminosa seja refletida, parte difratada e outra absorvida. O ângulo de difração é inversamente proporcional ao tamanho da gota, tornando-se assim possível obter a distribuição do tamanho de gotas em um spray. O modelo de distribuição de tamanho de gotas ajustado aos dados experimentais foi o de Rosin-Rammler, por ser o mais adequado para distribuição gotas no interior de lavadores Venturi (Guerra et al., 2009; Guerra, 2009). Os valores dos parâmetros do modelo de Rosin-Rammler (espalhamento $(n)$ e diâmetro médio $(\bar{D})$ ) foram obtidos através de ajuste para posterior utilização nas simulações. Para avaliar a influência do diâmetro médio do modelo de Rosin-Rammler nas simulações, para a condição de injeção por 2 orífícios e $\mathrm{Vg}=64 \mathrm{~m} / \mathrm{s}(\mathrm{E}-2)$, variou-se os valores do diâmetro médio do modelo $(\bar{D})$ em $\pm 20 \%$ do valor ajustado com base no valor experimental.

O acoplamento pressão-velocidade foi solucionado através do método SIMPLE, utilizando-se discretizações Upwind de segunda-ordem para as equações do movimento e de turbulência (Fluent, 2011). Efeitos gravitacionais foram desprezados nas simulações. Os critérios de convergência para todas variáveis do problema foram mantidos em $10^{-4}$, com parâmetros de relaxação na faixa de 0,2 a 0,4. A malha computacional foi elaborada com o software GAMBIT 2.4.6, a partir de um teste de independência de malha, respeitando-se as dimensões e demais características geométricas do equipamento. A malha híbrida adotada neste trabalho foi composta por 1.178 .610 células, contando com 2.540.298 faces e 333.383 nós. Células hexaédricas foram utilizadas nas regiões de entrada e saída da garganta do equipamento e células tetraédricas na região da garganta, que por sua vez continham os orifícios de injeção de líquido.

\section{RESULTADOS E DISCUSSÕES}

A Figura 4 apresenta a distribuição de tamanho de gotas medida experimentalmente e a ajustada com o modelo de distribuição de Rosin-Rammler. Como pode ser observado na Figura 4, o modelo de 


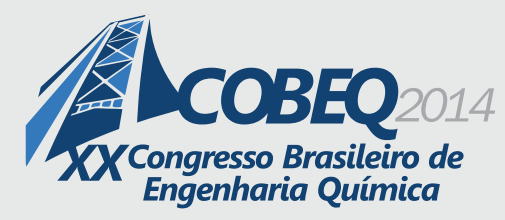

distribuição de Rosin-Rammler se ajustou adequadamente aos dados experimentais. Através do ajuste foi possível determinar dois parâmetros do modelo de distribuição de Rosin-Rammler para cada condição experimental, como mostrado na Tabela 2.



(a)



(b)

Figura 4 - Distribuição de tamanho de gotas experimental e por Rosin-Rammler: (a) E-1; (b) E-2.

Tabela 2 - Parâmetros do modelo de distribuição de Rosin-Rammler

\begin{tabular}{|c|c|c|}
\hline Experimento & $\bar{D}(\mu \mathrm{m})$ & $\mathrm{n}$ \\
\hline E-1 & 190 & 2 \\
\hline E-2 & 202 & 1,9 \\
\hline E-3 & 160 & 1,9 \\
\hline
\end{tabular}

A Figura 5 apresenta a distribuição de concentração da fase dispersa (líquido) simuladas, em $\mathrm{kg} / \mathrm{m}^{3}$, para as diferentes condições experimentais avaliadas neste trabalho. Na Figura 5 pode-se verificar que ao adentrar a garganta do lavador a fase dispersa apresenta uma curvatura em virtude das forças de arraste do gás. Tal comportamento é condizente com os resultados experimentais obtidos por Guerra et al. (2012), Gonçalves et al. (2004) e Gonçalves (2000). Comparando-se os resultados dos experimentos E-2 e E-3, é possível verificar que para a condição de menor velocidade do gás (E2) o encontro das gotas ocorreu em um ponto mais próximo a injeção e em maior concentração. Nesse caso, além da força de arrasto ser menor, as gotas medidas foram maiores, apresentando maior inércia e tendência a seguir a trajetória de injeção, quando comparadas ao caso E-3.

Na figura 6 são apresentados os resultados simulados de fluxo normalizados pela concentração média em um plano localizado a 1,6 cm do ponto de injeção de líquido, posição onde os dados experimentais foram obtidos no equipamento. È possível verificar que a dispersão do líquido experimental e simulada apresentaram comportamento simulares para os experimentos E-1 e E3. Para o experimento E-1 (Figura 6a) observa-se um único pico com alto fluxo de gotas na região central da garganta. No caso E-3 (Figura 6c) pode-se verificar dois picos acentuados com alto 


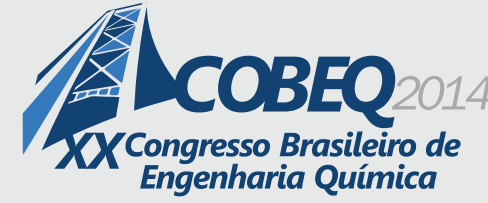

19 a 22 de outubro de 2014

Florianópolis/SC

fluxo de líquido. Já no experimento E-2 (Figura 6b) o comportamento e os valores dos fluxos normalizados de líquido medidos experimentalmente ficaram bem distantes do simulado. Como pode ser visto na Figura 5b e confirmado na Figura 6b, nessas condições as simulações previram maior fluxo de líquido na região central da garganta ocasionado pelo encontro frontal das gotas.

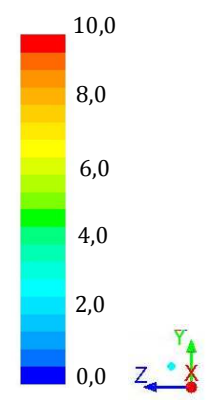

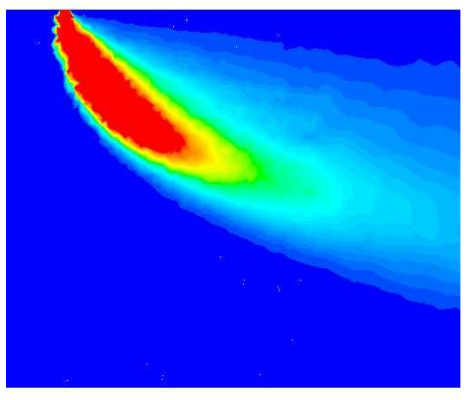

(a)



(b)



(c)

Figura 5 - Contornos de concentração $\left(\mathrm{kg} / \mathrm{m}^{3}\right)$ da fase dispersa (a) E-1 (b) E2 (c) E-3
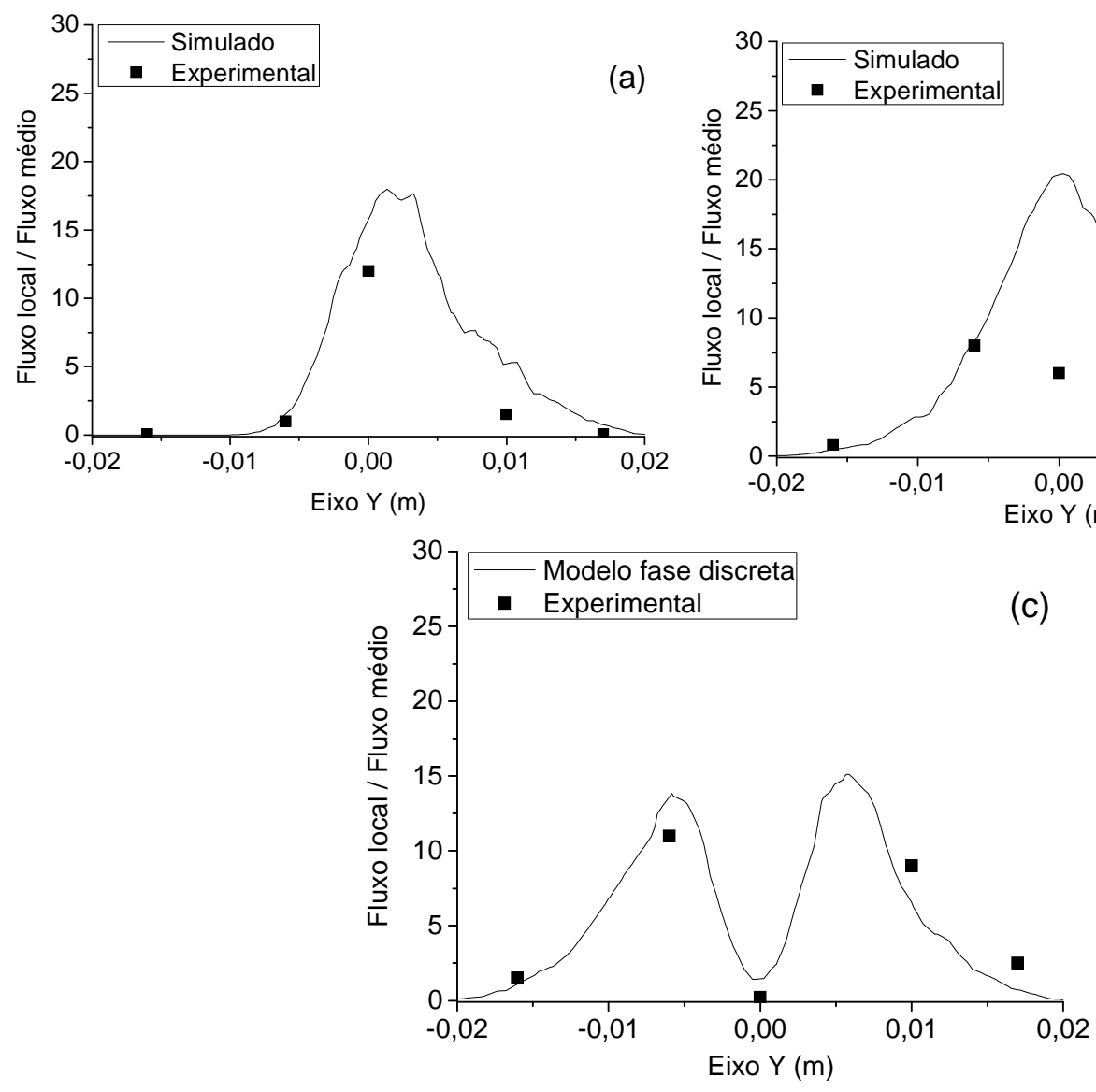

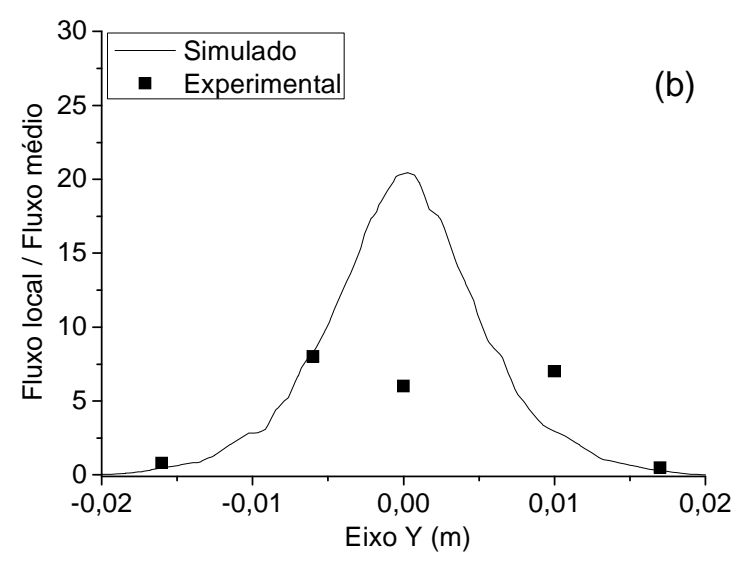

(c)

Figura 6 - Perfis de dispersão de líquido (a) E-1 (b) E-2 (c) E-3. 
Com o objetivo de verificar a influência do diâmetro médio do modelo de Rosin-Rammler na dispersão de líquido simulada, foram efetuadas simulações da dispersão de líquido considerando valores em um intervalo $\pm 20 \%$ para $\bar{D}$, no experimento E-2. Estes resultados são apresentados na Figura 7.
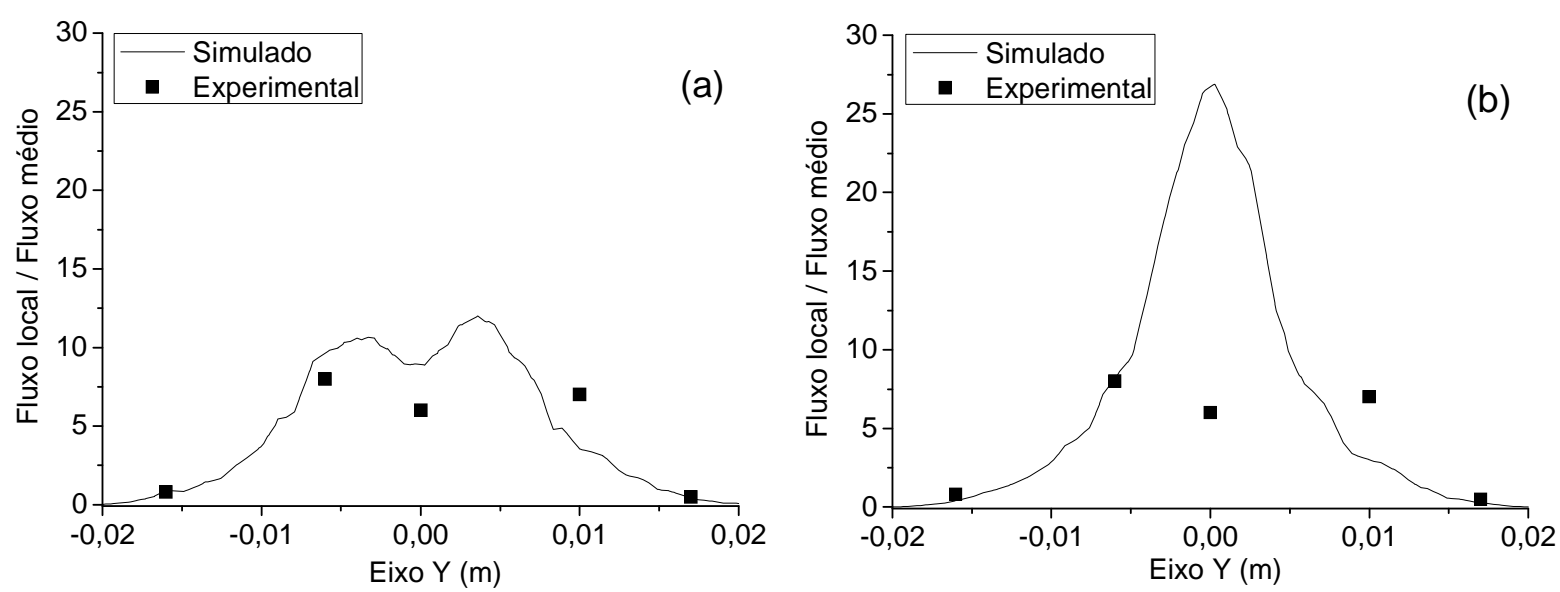

Figura 7 - Comparação dos perfis de dispersão de líquido experimental (E-2) e simulado, variando o diâmetro médio do modelo de Rosin-Rammler: (a) $\bar{D}=194 \mu \mathrm{m}$; (b) $\bar{D}=242 \mu \mathrm{m}$.

A Figura 7 a apresenta o perfil de distribuição de líquido com $\bar{D}=194 \mu \mathrm{m}$ e a Figura $7 \mathrm{~b}$ com $\bar{D}=242 \mu \mathrm{m}(-20 \%$ e +20 no valor de $\bar{D}$, respectivamente $)$ para o caso E-2. Comparando-se as Figuras $6 \mathrm{~b}$ e 7 , é possível verificar que alterações no diâmetro médio ocasionaram diferenças na concentração de gotas. Comparando-se a Figura $6 \mathrm{~b}$ e $7 \mathrm{~b}$ é possível uma diferença marcante na concentração de gotas na região central do equipamento, onde um maior diâmetro médio resultou em um aumento de 20 para 26 no valor da concentração máxima, indicando que a inércia das gotas influência na dispersão do líquido. A diminuição no tamanho da gota em $20 \%$, apresentada na Figura $7 \mathrm{a}$, fez com que a dispersão do líquido simulada se aproximasse ainda mais da experimental. Nesse caso, o fluxo de líquido normalizado apresentou dois picos suaves na região central do lavador. Tais resultados indicam que o tamanho médio da gota influência significativamente no comportamento de distribuição de líquido no interior de um lavador Venturi.

\section{CONCLUSÕES}

O modelo lagrangeano de fase discreta mostrou-se uma opção adequada para a simulação da distribuição de gotas no interior do lavador Venturi. Para o caso em que os resultados simulados apresentaram maiores desvios em relação ao experimental a avaliação da influência do diâmetro médio da gota indicou que os resultados são significativamente sensíveis a este parâmetro. Variações de mais ou menos $20 \%$ no valor do diâmetro médio alteraram significativamente a dispersão qualitativa e quantitativa do líquido dentro da garganta do lavador Venturi. Observou-se que o comportamento físico da dispersão de líquido manteve-se conservado com as variações de diâmetro de gota, pois maiores gotas resultaram em maior inércia e consequentemente maiores concentrações na região central. 


\section{REFERÊNCIAS}

ANANTHANARAYANAN, N. V.; VISWANATHAN, S. Effect of nozzle arrangement on Venturi scrubber performance. Ind. Eng. Chem. Res. v. 38, p. 4889-4900, 1999.

EKMAN, F. O.; JOHNSTONE, H. F. Collection of aerosols in a Venturi scrubber. Ind. and Eng. Chem. v. 43, p. 1358-1363, 1951.

FLUENT 14.0 Theory Guide. Fluent Inc.: Lebanon, NH, 2011.

GONÇALVES, J.A.S. Aspectos da modelagem matemática de lavadores Venturi. Tese de doutorado, Universidade Federal de São Carlos, São Carlos - Brasil, 2000.

GONÇAlveS, J. A. S., COSTA, M. A. M.; AGUiAR, M. L.; COURY, J. R. Atomization of liquids in a Pease-Anthony Venturi scrubber - Part II. Droplet dispersion. J. Haz. Mat. p. 147$157,2004$.

GUERRA, V. G.; GONÇALVES, J. A. S.; COURY, J. R. Experimental investigation on the effect of liquid injection by multiple orifices in the formation of droplets in a Venturi scrubber. $J$. Haz. Mat., v.161, p.351-359, 2009.

GUERRA, V. G . Investigação do efeito da injeção de líquido por orifícios múltiplos na formação de gotas em um lavador Venturi. 150p. Tese (Doutorado em Engenharia Química) Universidade Federal de São Carlos, São Carlos, 2009.

GUERRA, V.G. ; BETTEGA, R. ; GONCALVES, J. A. S. ; COURY, J. R. . Pressure Drop and liquid distribution in a Venturi Scrubber: Experimental data and CFD simulation. Ind. \& Eng. Chemistry Research, v. 51, p. 8049-8060, 2012.

VISWANATHAN, S.; GNYP, A. W.; ST. PIERRE, C. C. Examination of gas flow in a Venturi scrubber. Ind. and Eng. Chem. Fund. v. 23, p. 303-308, 1984 